\title{
Síndrome de Burnout y su impacto en los trabajadores de las pymes del sector alimenticio de la ciudad de Guayaquil, Ecuador
}

\author{
Burnout Syndrome and its impact on workers of the food \\ industry's SMEs of the city of Guayaquil, Ecuador
}

\author{
Román Tinoco Isabel Mishelle ${ }^{1}$ \\ isatiroman@gmail.com \\ https://orcid.org/0000-0003-2218-8529 \\ Muñoz Loor Karina ${ }^{2}$ \\ kmunoz@uteg.edu.ec \\ https://orcid.org/0000-0001-9767-2783
}

Recibido: 01/07/2020, Aceptado: 01/09/2020

\section{RESUMEN}

El Síndrome de Burnout (SB) es el resultado del desgaste emocional, falta de realización personal y despersonalización de los empleados de empresas públicas y privadas, comprometiendo la productividad, eficiencia y competitividad comercial institucional, así como su crecimiento. Este estudio, de tipo cuantitativo de corte transversal y análisis descriptivo, utilizó 361 trabajadores de las pymes del sector alimenticio en la ciudad de Guayaquil. Como instrumento se empleó una encuesta auto-administrada, compuesta por diez preguntas cerradas con una escala de Likert de 5-puntos, basado en el cuestionario de Maslach Burnout Inventory. Como resultado, se evidenció que los empleados de las empresas, no reciben charlas motivacionales que les permitan potencializar la eficacia y eficiencia de su desempeño laboral. Se desarrolló un análisis actual sobre las pymes de este sector, verificando como su capacidad de crecimiento ha sido lenta, y esto se debe en gran parte a la falta de dedicación de los empleados. Finalmente, en la propuesta se desarrolló un programa de incentivos laborales que sirven para poder minimizar los efectos del SB.

Palabras clave: Síndrome de Burnout, Maslach Burnout Inventory, pymes, trabajadores, sector alimenticio

\section{ABSTRACT}

Burnout Syndrome (BS) is a state of emotional, mental and physical exhaustion among employee from public and private institutions, compromising productivity, efficiency and commercial competitivity of enterprise such as its commercial growth. This quantitative study called cross sectional study included a descriptive analysis. This study recruited 361 workers from small and middle enterprises (SMEs) with commercial activities associated with the food industry, in Guayaquil, Ecuador. As the

\footnotetext{
${ }^{1}$ Universidad Tecnológica Empresarial de Guayaquil, Ecuador

2 Universidad Tecnológica Empresarial de Guayaquil, Ecuador.
} 


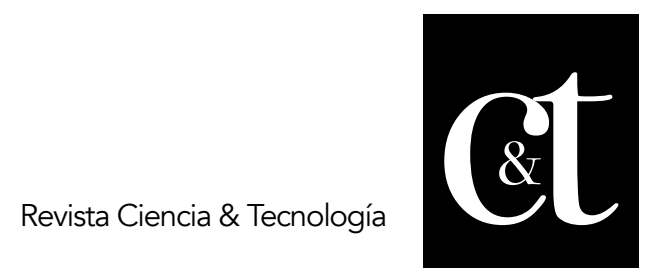

No. 28,31 de octubre de 2020

ISSN impreso: 1390 - 6321

ISSN online: 2661 - 6734

study instrument, a questionnaire was applied among the employees. Such instrument used a self-designed and close-based questions, which included a 5-points Likert scale-based on Maslach Burnout Inventory Questionnaire. Evidences suggested that the efficiency and efficacy of employees' performances are needed to being potentialized by self-motivational programs which are absent among companies. An analysis has been carried out on SMEs in this sector, verifying that companies' growth capacity have been slowed down and this is due to a great extent to the lack of dedication of employees. Finally, the suggestion of an interventional program is needed among workers such as work incentives to minimize the effects of BS.

Keywords: Burnout Syndrome, Maslach Burnout Inventory, SMEs, workers, food industry

\section{Introducción}

Un estudio desarrollado en Ecuador, reveló que el $70 \%$ los trabajadores institucionales presentan agotamiento al final de la jornada laboral (Soledispa, 2016; Velasco y Noriega, 2006).

El Síndrome de Burnout (SB) se ha definido como una respuesta crónica al estrés laboral entre profesionales que trabajan en las organizaciones de servicios de atención (Agudelo et al., 2011; Amutio, Ayestaran y Smith, 2008). Según Maslach y Jackson (1982), una respuesta de este tipo es un proceso caracterizado por baja realización personal (auto-evaluación negativa) y de alta agotamiento emocional (por contacto con personas) y despersonalización (cognitivo-aptitudinal o sentimientos negativos o disminución de la autoestima). El SB afecta la capacidad laboral y la relación con las demás personas de la empresa (Aranda y Pando, 2015; Camacho y Arias, 2015; Menghi y Oros, 2014). Es un estado de agotamiento físico, emocional y mental causado por la preocupación a largo plazo lo que conduce a un deterioro en la calidad de los servicios prestados por la organización. Adicionalmente, este síndrome está asociado con enfermedades coronarias o cardiopatía isquémicas, hipertensión, enfermedad péptica o colitis nerviosa (Aranda y Pando, 2015; Moreno y Báez, 2010; Guillén, 2006; Ortega, Salas y Correa, 2011; Velasco y Noriega, 2006).

Entre las variables identificadas como antecedentes del SB en profesionales, se encuentran el estilo sociodemográfico conflictivo, la ambigüedad de rol, la falta de apoyo social en el trabajo, la autonomía, y sobre todo el aporte de los directivos (Minchola, González y Terán, 2013; CEPAL, 2013; Ortega, Salas y Correa, 2011).

Aunque el SB ha sido estudiado como resultado de variables organizacionales, tales como el estrés de rol, la sobrecarga de trabajo o las relaciones interpersonales; una serie de variables personales, tales como capacidades, las percepciones y los factores de la personalidad están íntimamente correlacionada con el síndrome (López, Marín y Alcalá, 2012; Mora, 2003).

Este estudio se desarrolló pensando en las necesidades de entidades pymes que buscan crecer económicamente, mejorar su productividad, pero sobre todo tratando de ofrecer un mejor servicio al cliente (Henrich y Rojas, 2013; Calvo, 2008). Las pymes que trabajan en el sector alimenticio generan un tenso ambiente laboral, en el cual, como consecuencia del estrés relacionado con el trabajo prolongado y extenso, puede generar alteraciones en el desempeño laboral, así como agotamiento 


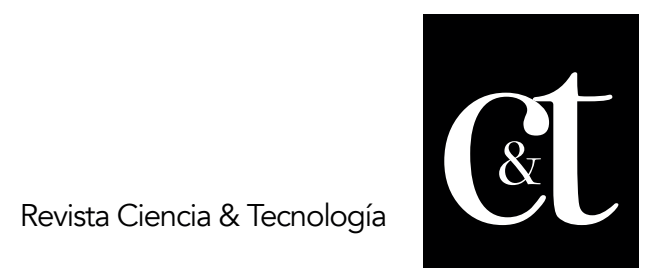

No. 28,31 de octubre de 2020

ISSN impreso: 1390 - 6321

ISSN online: 2661 - 6734

de los recursos físicos y emocionales del individuo (Aranda y Pando, 2015; Chiang y Sigoña, 2014; Camacho y Arias, 2015).

Sin embargo, Ortega, Salas y Correa (2011), reconocen que muchos de los directivos desconocen el SB (deterioro profesional) y su impacto en el rendimiento laboral en los procesos de generación y producción, así como la relación con sus clientes externos al no satisfacer sus necesidades frente a la misión y visión de la institución (Camacho y Arias, 2015; Molina, López y Contreras, 2014; Organización Internacional del Trabajo, 2016).

Gran cantidad de pymes buscan el crecimiento comercial, además de que sus productos y marcas sea altamente competitivas (Piedrahita, 2014; Rodríguez, Martínez y López, 2013). Esto conlleva a que los directivos exijan mucho más de sus trabajadores, y buscan que se cumpla todos los procesos empresariales con alta rigurosidad (Carrasco, 2015; Rodríguez y Pérez, 2011; Rodríguez y Pérez 2014).

Durante años, la satisfacción del empleado ha sido un área clave de la investigación entre los psicólogos industriales y organizacionales. Hay razones importantes por las que las empresas deben preocuparse por la satisfacción laboral de los empleados, que se pueden clasificar de acuerdo con el enfoque en el empleado o la organización (Alles, 2017; Rodríguez, Pérez y Montero, 2012). Los empleados satisfechos son una condición previa para aumentar la productividad, capacidad de respuesta, la calidad y el servicio al cliente (Alles, 2013; Rodríguez, Pérez y Montero, 2012).

\section{Desarrollo}

Este estudio transversal, descriptivo, de datos primarios recolectados por medio de una encuesta auto-administrada con preguntas cerradas basados en el cuestionario de Maslash Burnout Inventory con respuestas según la escala de Likert de 5 puntos, llevada a cabo en empresas privadas del sector alimenticio, realizada entre el mes de abril a junio del año 2018. La encuesta fue validada por 2 especialistas en psicología (Dra. Elena Soledispa y Dr. Alex Verdesoto) y se obtuvo el consentimiento informado de los participantes.

Para el muestreo se realizó seleccionando entre los empleados de las 13.426 PYMES dedicadas a la manufactura, de estas el $45 \%$ (6.042 pymes/un solo trabajador por cada empresa) son del sector alimentario en la ciudad de Guayaquil, que realizan actividades como bebidas, chocolates y confites, molinería, panadería, lácteos, aceites y productos cárnicos.

El muestreo se lo determinó aplicando la fórmula:

$$
\mathbf{n}=\frac{Z^{2}+N+P+Q}{\left(\left(e^{2}(\mathbb{N}-1)\right)+\left(Z^{2}+P+0\right)\right.}
$$

En donde, $Z=$ Nivel de confianza (1.96); $e=$ Margen de error $(0.05) ; p=$ Probabilidad de éxito $(0.5) ; \mathrm{q}=$ Probabilidad de fracaso $(0.5) ; \mathrm{N}=$ Total de la población; $\mathrm{Z}=$ Nivel de confianza (1.96); $e=$ Margen de error $(0.05) ; p=$ Probabilidad de éxito $(0.5) ; q=$ Probabilidad de fracaso $(0.5)$ y $\mathrm{N}=$ Total de la población. Obteniéndose una muestra de 361 trabajadores para este estudio. 
Para el análisis estadístico se empleó el programa estadístico IBM-SPSS v22, para analizar las variables categóricas ordinales mediante el análisis de frecuencia y frecuencia acumulada, así como la proporción de cada categoría o ítem.

\section{Resultados}

1.- ¿Se siente usted agotado y sin ganas de hacer nada?

\section{Tabla 1. Agotamiento emocional}

\begin{tabular}{lll}
\hline Características & $\begin{array}{l}\text { Frecuencia } \\
\text { Absoluta }\end{array}$ & $\begin{array}{l}\text { Frecuencia } \\
\text { Relativa }\end{array}$ \\
\hline Nunca & 10 & $3 \%$ \\
Casi nunca & 25 & $7 \%$ \\
A veces & 66 & $17 \%$ \\
Casi siempre & 173 & $45 \%$ \\
Siempre & 110 & $29 \%$ \\
Total & 384 & $\mathbf{1 0 0} \%$ \\
\hline
\end{tabular}

Fuente: Elaboración propia en base a resultados de encuesta

El $45 \%$ y el $29 \%$ de los encuestados denotaron que sienten un cansancio emocional de forma recurrente, lo que ha generado en ellos el desgano por hacer las cosas

2.- ¿Tiene usted pensamientos negativos sobre el lugar donde trabaja?

\section{Tabla 2. Aparición de pensamientos negativos}

\begin{tabular}{lll}
\hline Caracteristicas & $\begin{array}{l}\text { Frecuencia } \\
\text { Absoluta }\end{array}$ & $\begin{array}{l}\text { Frecuencia } \\
\text { Relativa }\end{array}$ \\
\hline Nunca & 10 & $3 \%$ \\
Casi nunca & 25 & $7 \%$ \\
A veces & 178 & $46 \%$ \\
Casi siempre & 136 & $35 \%$ \\
Siempre & 35 & $9 \%$ \\
Total & $\mathbf{3 8 4}$ & $\mathbf{1 0 0 \%}$ \\
\hline
\end{tabular}

Fuente: Elaboración propia en base a resultados de encuesta

El $46 \%$ de los encuestados siendo la parte mayoritaria, ha denotado que a veces tienen pensamientos negativos en su jornada laboral, además de que el $35 \%$, siendo la otra parte de mayor cantidad, especificó que lo tiene casi siempre.

3.- ¿Es usted indiferente y no hace un esfuerzo por comprender a las personas que lo rodean? 


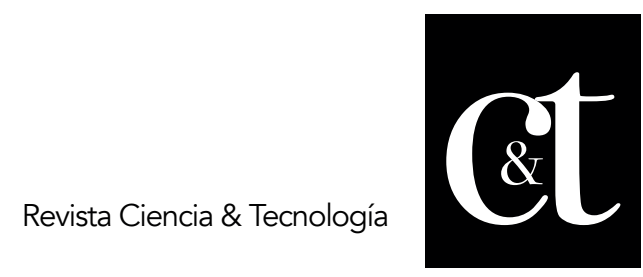

No. 28,31 de octubre de 2020

ISSN impreso: 1390 - 6321

ISSN online: 2661 - 6734

Tabla 3. Indiferencia personal

\begin{tabular}{lll}
\hline Caracteristicas & $\begin{array}{l}\text { Frecuencia } \\
\text { Absoluta }\end{array}$ & $\begin{array}{l}\text { Frecuencia } \\
\text { Relativa }\end{array}$ \\
\hline Nunca & 85 & $22 \%$ \\
Casi nunca & 52 & $14 \%$ \\
A veces & 73 & $19 \%$ \\
Casi siempre & 110 & $29 \%$ \\
Siempre & 64 & $17 \%$ \\
Total & 384 & $100 \%$ \\
\hline
\end{tabular}

Fuente: Elaboración propia en base a resultados de encuesta

Referente a la indiferencia personal, el $29 \%$ mencionó que lo hace casi siempre, es decir, su accionar frecuente radica en enfrascarse en sus problemas y no tener que desviarse por los de los demás.

4.- ¿Se irrita con facilidad ante los problemas pequeños?

Tabla 4. Irritación con facilidad

\begin{tabular}{lll}
\hline Caracteristicas & $\begin{array}{l}\text { Frecuencia } \\
\text { Absoluta }\end{array}$ & $\begin{array}{l}\text { Frecuencia } \\
\text { Relativa }\end{array}$ \\
\hline Nunca & 4 & $1 \%$ \\
Casi nunca & 33 & $9 \%$ \\
A veces & 58 & $15 \%$ \\
Casi siempre & 100 & $26 \%$ \\
Siempre & 189 & $49 \%$ \\
Total & $\mathbf{3 8 4}$ & $\mathbf{1 0 0 \%}$ \\
\hline
\end{tabular}

Fuente: Elaboración propia en base a resultados de encuesta

Conforme a los datos se puede notar como el $49 \%$ de los encuestados ha manifestado que siempre se irritan frente a los problemas pequeños, mientras que la otra parte mayoritaria es el $26 \%$ denotando casi siempre.

5.- ¿Siente que no lo valoran, ni reconocen su esfuerzo en el trabajo? 
Tabla 5. Poca valoración

\begin{tabular}{lll}
\hline Caracteristicas & $\begin{array}{l}\text { Frecuencia } \\
\text { Absoluta }\end{array}$ & $\begin{array}{l}\text { Frecuencia } \\
\text { Relativa }\end{array}$ \\
\hline Nunca & 18 & $5 \%$ \\
Casi nunca & 11 & $3 \%$ \\
A veces & 66 & $17 \%$ \\
Casi siempre & 141 & $37 \%$ \\
Siempre & 148 & $39 \%$ \\
Total & 384 & $100 \%$ \\
\hline
\end{tabular}

Fuente: Elaboración propia en base a resultados de encuesta

El $39 \%$ denotado en la variable siempre, y el $37 \%$ especificado en la variable casi siempre, demuestra que los trabajadores mantienen esa poca atención que los hace vulnerables ante los problemas que puedan surgir.

6.- ¿Siente que nadie lo escucha o le pone algo de atención a sus requerimientos y preocupaciones?

Tabla 6. Poca atención

\begin{tabular}{lll}
\hline Caracteristicas & $\begin{array}{l}\text { Frecuencia } \\
\text { Absoluta }\end{array}$ & $\begin{array}{l}\text { Frecuencia } \\
\text { Relativa }\end{array}$ \\
\hline Nunca & 25 & $7 \%$ \\
Casi nunca & 25 & $7 \%$ \\
A veces & 25 & $7 \%$ \\
Casi siempre & 110 & $29 \%$ \\
Siempre & 199 & $52 \%$ \\
Total & 384 & $100 \%$ \\
\hline
\end{tabular}

Fuente: Elaboración propia en base a resultados de encuesta

Como se puede notar, el $52 \%$ de los encuestados manifestaron que siempre sienten esa poca atención así también los estipularon el $29 \%$ a través del casi siempre. Esto demuestra que sus requerimientos se encuentran pasando desapercibidos en el medio local.

7.- ¿Se siente presionado para cumplir sus metas? 
Tabla 7. Presión para cumplir metas

\begin{tabular}{lll}
\hline Caracteristicas & $\begin{array}{l}\text { Frecuencia } \\
\text { Absoluta }\end{array}$ & $\begin{array}{l}\text { Frecuencia } \\
\text { Relativa }\end{array}$ \\
\hline Nunca & 21 & $5 \%$ \\
Casi nunca & 34 & $9 \%$ \\
A veces & 34 & $9 \%$ \\
Casi siempre & 140 & $36 \%$ \\
Siempre & 155 & $40 \%$ \\
Total & $\mathbf{3 8 4}$ & $\mathbf{1 0 0 \%}$ \\
\hline
\end{tabular}

Fuente: Elaboración propia en base a resultados de encuesta

De acuerdo a la figura, se puede notar como el $40 \%$ de los encuestados mencionaron que siempre tienen esa presión para poder cumplir con sus metas, lo que demuestra que existe esa frustración cuando no se cumplen con las mismas.

8.- ¿Siente que está en el trabajo correcto?

Tabla 8. Satisfacción con el trabajo

\begin{tabular}{lll}
\hline Características & $\begin{array}{l}\text { Frecuencia } \\
\text { Absoluta }\end{array}$ & $\begin{array}{l}\text { Frecuencia } \\
\text { Relativa }\end{array}$ \\
\hline Nunca & 124 & $32 \%$ \\
Casi nunca & 111 & $29 \%$ \\
A veces & 54 & $14 \%$ \\
Casi siempre & 66 & $17 \%$ \\
Siempre & 29 & $8 \%$ \\
Total & 384 & $100 \%$ \\
\hline
\end{tabular}

Fuente: Elaboración propia en base a resultados de encuesta

Conforme a los datos se puede notar como el $32 \%$ de los encuestados manifestaron que nunca han pensado que están en el trabajo correcto, lo que demuestra que laboran por la necesidad más no por satisfacción.

9.- ¿Se siente frustrado por algunas situaciones que suceden en el trabajo? 


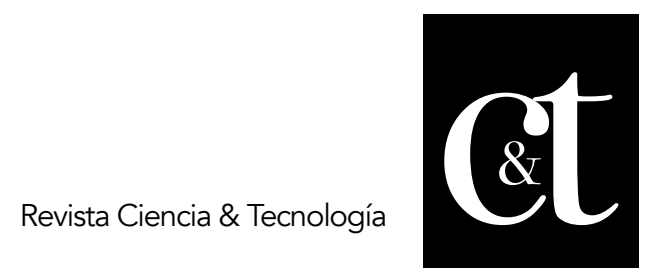

No. 28,31 de octubre de 2020

ISSN impreso: 1390 - 6321

ISSN online: 2661 - 6734

Tabla 9. Influencia en las personas

\begin{tabular}{lll}
\hline Caracteristicas & $\begin{array}{l}\text { Frecuencia } \\
\text { Absoluta }\end{array}$ & $\begin{array}{l}\text { Frecuencia } \\
\text { Relativa }\end{array}$ \\
\hline Nunca & 41 & $11 \%$ \\
Casi nunca & 68 & $18 \%$ \\
A veces & 81 & $21 \%$ \\
Casi siempre & 94 & $24 \%$ \\
Siempre & 100 & $26 \%$ \\
Total & $\mathbf{3 8 4}$ & $\mathbf{1 0 0 \%}$ \\
\hline
\end{tabular}

Fuente: Elaboración propia en base a resultados de encuesta

Según los datos de la figura, se puede notar como el $26 \%$ de los encuestados manifestaron que se sienten frustrados cuando las cosas internas no están funcionando, o no ocurren como están proyectadas, causando malestar.

10.- ¿Siente que le falta tiempo para hacer lo planeado?

Tabla 10. Falta de tiempo

\begin{tabular}{lll}
\hline Caracteristicas & $\begin{array}{l}\text { Frecuencia } \\
\text { Absoluta }\end{array}$ & $\begin{array}{l}\text { Frecuencia } \\
\text { Relativa }\end{array}$ \\
\hline Nunca & 55 & $14 \%$ \\
Casi nunca & 35 & $9 \%$ \\
A veces & 85 & $22 \%$ \\
Casi siempre & 98 & $26 \%$ \\
Siempre & 111 & $29 \%$ \\
Total & 384 & $100 \%$ \\
\hline
\end{tabular}

Fuente: Elaboración propia en base a resultados de encuesta

De acuerdo a los datos se puede notar como el $29 \%$ de los encuestados estipularon que siempre sienten esa necesidad de tiempo, es decir, que les hace falta para poder logara sus metas y propósitos.

\section{Conclusiones}

Se diagnosticaron las causas del SB en los trabajadores de las pymes del sector alimenticio de la ciudad de Guayaquil, donde se pudo corroborar que existen diversos factores tales como el clima laboral, la falta de interés por parte de los directivos, la falta de incentivos y también el hecho de que no se ha evaluado si el trabajador se siente conforme con su puesto laboral actual.

Se evaluaron las medidas que han tomado los directivos de las pymes del sector alimenticio de la ciudad de Guayaquil, las cuales han sido casi nulas, puesto que las estrategias aplicadas han sido los agasajos al finalizar el año y brindar los beneficios sociales, que en realidad son el derecho de todo trabajador. 


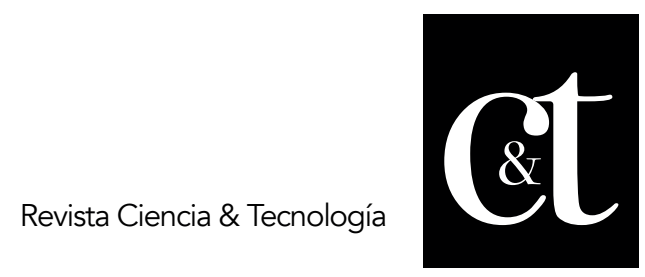

No. 28,31 de octubre de 2020

ISSN impreso: 1390 - 6321

ISSN online: 2661 - 6734

Se determinaron los efectos del SB la productividad y desempeño laboral de los trabajadores de las pymes del sector alimenticio de la ciudad de Guayaquil, donde se pudo notar que ellos sienten ese desinterés de dar lo mejor, es decir, observan y tratan a su trabajo como algo rutinario.

Se sugiere desarrollar un plan de capacitación para reducir los efectos del SB en los empleados de las pymes del sector alimenticio, este plan debe enfocarse en incentivos, así como capacitaciones, control de estrés, y relaciones interpersonales.

Desarrollar un plan de relación interna para que exista una conexión entre los empleados y los directivos, de esta forma los trabajadores se sienten más atendidos y mantengan un compromiso con la empresa, de esa forma se puede asegurar que el desempeño laboral sea más eficaz y eficiente.

\section{Referencias bibliográficas}

Agudelo, C.A., Castaño, J.J., Arango, C.A., Durango, L.A., Muñoz, V., Ospina, A., Ramírez, J., Salazar, N., Serna, J.D. y Taborda, J. (2011). Prevalencia y factores psicosociales asociados al Síndrome de Burnout en médicos que laboran en instituciones de las ciudades de Manizales y la Virginia (Colombia). Archivos de Medicina (Col), vol. 11, núm. 2, julio-diciembre, 2011, pp. 91-100

Alles, M. (2013). Construyendo Talento: Programas de desarrollo para el crecimiento de las personas y la continuidad organizacional. Barcelona: Granica. ISBN 978950-641-548-8. Recuperado de: https://jgestiondeltalentohumano.files.wordpress.com/2013/11/construyendotalento-programas-de-desarrollo-para-el-crecimiento-de-las-personas-y-lacontinuidad-de-las-organizaciones-martha-alles.pdf [2/9/2019]

Alles, M. (2017). Dirección estratégica de Recursos Humanos. Gestión de Competencias. Casos. Bogotá: Granica. ISBN 978-950-641-490-0. Recuperado de

https://www.academia.edu/25666344/Direccion_estrategica_de_recursos_hum anos_-_Alles [25/8/2019]

Amutio, A., Ayestaran, S. y Smith, J. (2008). Evaluación del burnout y bienestar psicológico en los profesionales sanitarios del País Vasco. Rev Psicól Trab Organiz. 24(2):235-252. ISSN 1576-5962

Aranda-Beltrán, C. y Pando-Moreno, M. (2015). Edad, síndrome de agotamiento profesional (burnout), apoyo social y autoestima en agentes de tránsito. Rev Colomb Psiquiatr, 39(3): 510-522. ISSN 0034-7450

Calvo, F. (2008). Estudio descriptivo del síndrome de Burnout en una muestra de profesionales de enfermería en el área sur de Gran Canaria. Rev Ansied Estrés, 14(1): 101-113.

Camacho, C. y Arias, F. (2015). Análisis de la Salud y Burnout en Profesores Mexicanos. Rev Salud, 11 (3): 168-171.

CEPAL (2013). Estudio económico de América Latina y el Caribe 2013: tres décadas de crecimiento económico desigual e inestable. CEPAL. ISBN 9789212211121. Recuperado de: https://www.cepal.org/es/publicaciones/1085-estudioeconomico-america-latina-caribe-2013-tres-decadas-crecimiento-economico 


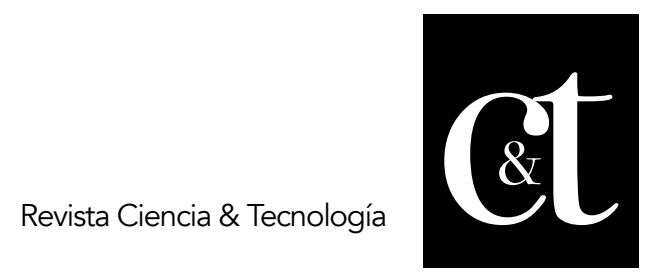

No. 28,31 de octubre de 2020

ISSN impreso: 1390 - 6321

ISSN online: 2661 - 6734

Chiang, M. y Sigoña, M. (2014). Burnout en trabajadores de hospitales comunitarios o de baja complejidad: una comparación de género y de nivel de escolaridad1. Rev Psicolog, 20(1): 32-52. ISSN 0716-8039.

Guillén, M. (2006). Ergonomía y la relación con los factores de riesgo en salud ocupacional. Rev Cubana Enfermería, 22(4): sep-dic. ISSN 0864-0319.

Henrich-Saavedra, M. y Rojas-Lazo, O. (2013). Aplicaciones de la metodología TRIZ en el diseño ergonómico de estaciones de trabajo. Rev Diseño Tecnolog, 16(1): 102- 107.

López-Torres, V., Marín-Vargas, M. y Alcalá-Álvarez, M. (2012). Ergonomía y Productividad: variables que se relacionan con la competitividad de las plantas. Ingeniería Industrial. Actualid Nuevas Tendencias, 3(9): 17-32.

Maslach, C. \& Jackson, S.E. (1982): Burnout in health professions: A social psychological analysis, en Sanders, G. y Suls, J. (Eds.). Social psychology of health and illness. Hillsdale, NJ: Erlbaum.

Menghi, M. y Oros, L. (2014). Satisfacción laboral y síndrome de burnout en docente de nivel primario. Rev Psicol, 10(20): 47-59.

Minchola-Gallardo, J., González-Veintimilla, F. y Terán-Iparraguirre, J. (2013). Riesgos ergonómicos en la salud de los trabajadores de un centro piscícola. Rev Scientia Agropecuar, 4(4): 303-311.

Molina, R., López, A. y Contreras, R. (2014). El emprendimiento y crecimiento de las Pymes. Acta Universitaria, vol. 24, núm. 1, diciembre, 2014, pp. 59-72.

Mora-Salas, M. (2003). El riesgo laboral en tiempos de globalización. Estudios Sociológicos, 21(63):643-666. Recuperado de: https://estudiossociologicos.colmex.mx/index.php/es/article/view/586/586 [15/8/2019]

Moreno-Jiménez, B. y Báez-León, C. (2010). Factores y riesgos psicosociales, formas, consecuencias, medidas y buenas prácticas. Madrid: Universidad Autónoma de Madrid. Recuperado de: http://comisionnacional.insht.es/InshtWeb/Contenidos/Documentacion/PUBLICA CIONES\%20PROFESIONALES/factores\%20riesgos\%20psico.pdf [1/8/2019]

Organización Internacional del Trabajo (OIT) (2016). Recurso humano $y$ productividad. ISSN 9789223311384. Suiza: OIT. Recuperado de: https://www.ilo.org/wcmsp5/groups/public/---ed_emp/---emp_ent/--ifp_seed/documents/instructionalmaterial/wcms_553925.pdf

Ortega, C., Salas, R. y Correa, R. (2011). Aspectos epidemiológicos del síndrome de burnout en el personal sanitario. Archiv Medic, 7(2): 4. doi: 10:3823/072.

Piedrahita, L.H. (2014). Algunas experiencias de la aplicación de la ergonomía en el sector minero. Rev Cienc Salud, 12 (especial): 69-76. Doi: http://dx.doi.org/10.12804/revsalud12.esp.2014.05.

Rodríguez, I., Martínez, M. y López, M. (2013). El riesgo percibido por el trabajador de la construcción: ¿qué rol juega el oficio? Rev Construc, 12(3):83-90.

Rodríguez Ruíz, Y. y Pérez Mergarejo, E. (2011). Ergonomía y simulación aplicadas a la industria. Ingenier Industr, 32(1): 2-11. 


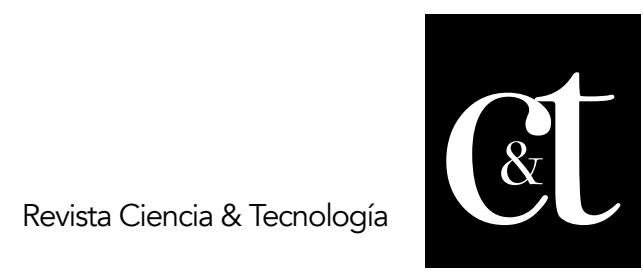

No. 28, 31 de octubre de 2020

ISSN impreso: 1390 - 6321

ISSN online: 2661 - 6734

Rodríguez Ruíz, Y. y Pérez Mergarejo, E. (2014). Procedimiento ergonómico para la prevención de enfermedades en el contexto ocupacional. Rev Cub Salud Púb, 40(2): 279-285.

Rodríguez Ruíz, Y., Pérez Mergarejo, E. y Montero Martínez, R. (2012a). Modelo de Madurez de Ergonomía para Empresas (MMEE). Rev Hombre Máquin, (40), 2230.

Soledispa, H. (2016). Reformas laborales estériles. Desarrollo Económico, No. 1. Instituto Ecuatoriano de Economía Política. Recuperado de: https://ieep.org.ec/wp-content/uploads/2019/10/DesarrolloEcono\%C3\%ACmico-N\%C2\%BA1-Abril-2016.pdf

Velasco-Tenorio, M. y Noriega-Elío, M. (2006). Evaluación de las causas de accidentes y enfermedades en una industria manufacturera de vidrio. Rev Salud Trabajador, 14(1):5-17. 